\title{
On Graver's Conjecture Concerning the Rigidity Problem of Graphs
}

\author{
Hiroshi Maehara \\ College of Education, Ryukyu University, \\ Nishihara, Okinawa, Japan
}

Abstract. We show that the four-dimensional case of Graver's conjecture is not true.

\section{Introduction}

A framework in Euclidean $d$-space $R^{d}$ is a graph whose vertices are points in $R^{d}$. A framework $F$ in $R^{d}$ is said to be $d$-flexible if we can continuously move the vertices of $F$ in $R^{d}$ so that

(1) the distance between adjacent vertices are kept unchanged, and

(2) at least a pair of vertices of $F$ change their mutual distance.

If a framework is not $d$-flexible, then it is said to be $d$-rigid.

Let $G$ be an abstract graph with $n$ vertices $1,2, \ldots, n$. Take $n$ points $p_{1}, \ldots, p_{n}$ in $R^{d}$, and define $p_{i}, p_{j}$ to be adjacent whenever $i, j$ are adjacent in $G$. Then we have a framework in $R^{d}$. This framework is denoted by $G(p)$, where $p=\left(p_{1}, \ldots, p_{n}\right) \in R^{n d}$. If all the coordinates of $p$ are algebraically independent over the rational field, then we call $G(p)$ a generic realization of $G$. The $d$-rigidity or $d$-flexibility of a generic realization $G(p)$ of $G$ is determined only by the abstract graph structure of $G$ and independent of the choice of $p$ (see, e.g., [1] and [5]). If a generic realization $G(p)$ of $G$ is $d$-rigid, then the graph $G$ is said to be generically $d$-rigid, otherwise it is said to be generically d-flexible.

Some techniques are proposed to build up a larger, generically $d$-rigid graph from a smaller one. The following two operations are valid:

(1) Addition of a new $d$-valent vertex.

(2) Exchange an edge $x y$ for a new $(d+1)$-valent vertex $z$ attached to $x, y$ and any other $d-1$ vertices.

These are known as Henneberg methods. Laman's theorem [4] states that every "edge-minimal" generically 2-rigid graph is obtained from $K_{2}=0-0$ by applying 

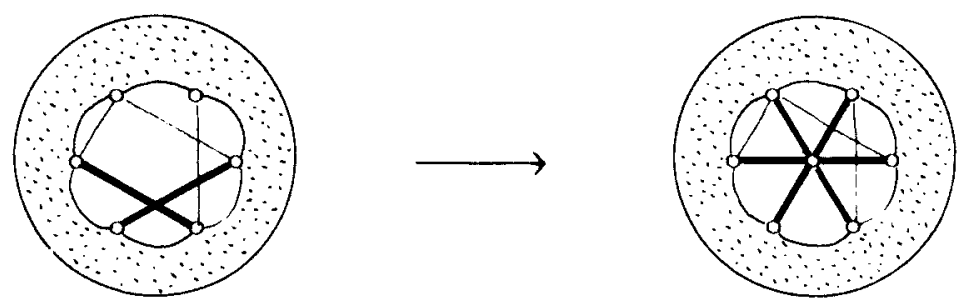

Fig. 1. Operation $X_{4}$.

operations (1) and (2). However, these are not sufficient for higher-dimensional cases. So other operations are sought.

Let $G$ be a graph with more than $d+1$ vertices. Consider the following operation $X_{d}$ on $G$ : add $G$ a new vertex $v$ and $d+2$ new edges connecting $v$ to some $d+2$ vertices of $G$, and remove any two nonadjacent edges among those $d+2$ vertices. The resulting graph is denoted by $X_{d} G$. Figure 1 shows the operation $X_{4}$.

Conjecture $1[6]$. If $G=(V, E)$ is a generically d-rigid graph with $|V| \geq d+2$ and $|E|=d|V|-d(d+1) / 2$, then $X_{d} G$ is also generically $d$-rigid.

This would be a simple consequence of the following Graver's conjecture (see Conjectures $4.7,4.8,5.5$, and 5.6 of [6]):

Conjecture 2 [3]. If $v$ is an $(n+2)$-valent vertex in $G$ adjacent to the vertex set $S$, $G-v$ is generically $n$-independent, $d(S, G-v) \geq 2$ and $d\left(S^{\prime}, G-v\right) \geq 1$ for all subsets of $n+1$ elements in $S$, then $G$ is generically $n$-independent.

For the definitions of "generic $n$-independence" and "the generic $n$-degree of freedom" $d(U, G)$ of a vertex set $U$ in a graph $G$, see, e.g., [6]. (We do not use these definitions in this paper.)

We prove

Theorem 1. There exists a generically 4-rigid graph $G=(V, E)$ with $|V| \geq 6$, $|E|=4|V|-10$, such that $X_{4} G$ is not generically 4-rigid.

Thus Conjecture 1 is not true, and hence the four-dimensional case of Graver's conjecture is also not true.

Preceding the proof, we state the following theorem, which is an easy corollary to Theorem 1 of [7].

Theorem 2. A complete bipartite graph $K_{m, n}\left(\neq K_{1,1}\right)$ is generically d-rigid if and only if

$$
m, n \geq d+1 \quad \text { and } m+n \geq(d+1)(d+2) / 2
$$

This theorem is essentially proved in [2], but they never stated it in this form. 


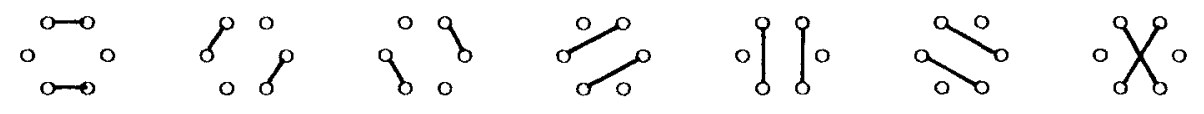

Fig. 2

Example. $K_{6,7}, K_{6,8}$, and $K_{7,7}$ are generically 4-flexible.

\section{Proof of Theorem 1}

Note that

$$
G_{0}:=K_{6}-(\text { an edge })
$$

is generically 4-rigid, since it is the "suspension" of $K_{4}$. The graph $G_{0}$ has 14 edges, and they can be partitioned into seven nonadjacent edge-pairs, see Fig. 2.

Consider the sequence of graphs

$$
G_{0}, G_{1}, \ldots, G_{7}
$$

where $G_{i}$ is obtained from $G_{i-1}$ by applying the operation $X_{4}$ : connecting the new vertex to the six vertices of $G_{i-1}$ that are inherited from $G_{0}$, and eliminating the $i$ th pair of edges in Fig. 2. Then $G_{7}$ is isomorphic to the complete bipartite graph $K_{6,7}$. However, $K_{6,7}$ is generically 4 -flexible by the above example. It is easy to see that in each $G_{i}$

$$
\text { (number of edges) }=4 \text { (number of vertices) }-10 \text {. }
$$

Hence one of $G_{i}(0 \leq i \leq 6)$ is a desired graph.

Remark. Professor W. Whiteley pointed out the following: The fifth graph $G_{5}\left(K(6,5)\right.$ plus four bars) is generically rigid, but the sixth graph $G_{6}(K(6,6)$ plus two bars) is generically flexible. The proofs rely on theorems in [7]. $K(6,6)$ plus two bars is infinitesimally flexible if all joints plus the two lines of the added bars lie on a quadric in 4-space-i.e., if 12 points plus the midpoints of the two added bars lie on a quadric. However, every 14 points in 4-space lie on a quadric. On the other hand, $K(6,5)$ plus four bars requires $11+4$ midpoints to lie on a quadric - and five points can be placed to avoid a quadric or the other degeneracies.

\section{Acknowledgment}

I would like to thank Professor W. Whiteley and the referee for valuable comments and suggestions. 


\section{References}

1 L. Asimov and B. Roth, The rigidity of graphs, Trans. Amer. Math. Soc. 245 (1978), 279-289.

2. E. D. Bolker and B. Roth, When is a bipartite graph a rigid framework?, Pacific J. Math. 90 (1980), $27-44$.

3. J. Graver, A combinatorial approach to infinitesimal rigidity, Preprint, Department of Mathematics, Syracuse University, Syracuse, NY 13210, 1984.

4. G. Laman, On graphs and rigidity of plane skeletal structures, J. Eng. Math. 4 (1970), 331-340.

5. B. Roth, Rigid and flexible framework, Amer. Math. Monthly 88 (1981), 6-21.

6. T. S. Tay and W. Whiteley, Generating an isostatic framework, Structural Topology 11 (1985), $21-69$.

7 W. Whiteley, Infinitesimal motions of a bipartite framework, Pacific J. Math. 110 (1984), 233-255.

Received February 2, 1989, and in revised form April 18, 1989. 